\title{
Effect of subinhibitory concentrations of antimicrobial agents on virulence factors of Shigella flexneri $2 a$ and Escherichia coli 0124
}

\author{
V. V. TETZ and LARISA L. NORMAN \\ Department of Microbiology, Virology and Immunology, Pavlov Medical Institute, 6/8 L. Tolstoy Str., \\ St Petersburg 197089, Russia
}

\begin{abstract}
Summary. The effect of subinhibitory concentrations of rifampicin, chloramphenicol, tetracycline and nalidixic acid on the interaction of Shigella flexneri 2a and Escherichia coli $\mathrm{O} 124$ with $\mathrm{HeLa}$ cells was examined. Antimicrobial agents altered adhesion, penetration and intracellular multiplication of the bacteria, as well as re-infection of adjacent cells. Chloramphenicol increased the virulence of the bacterial strains whereas nalidixic acid, tetracycline and rifampicin diminished it.
\end{abstract}

\section{Introduction}

Shigella spp. and enteroinvasive Escherichia coli are important causes of gastrointestinal disorders, especially in children. Antibiotic concentrations lower than the minimal inhibitory concentration produce a wide variety of effects on bacterial cells. ${ }^{1-4}$ The effect of subinhibitory concentrations of antibiotics on the host-parasite interaction may be important during the early stages of infection because the bacteria separate themselves from their external environment after colonisation by a surface film. ${ }^{5,6}$ Adhesion is the first stage of the host-parasite interaction and several studies have investigated the adhesion of different bacteria to epithelial cells in the presence of concentrations of antibiotics below the MIC.$^{1-4}$ However, the influence of low concentrations of antimicrobial agents on other stages of the bacteria-host interactions which precede colony formation remains practically unknown. The interactions between Shigella spp., enteroinvasive $E$. coli and host cells, which include adhesion, microbial entry into epithelial cells, intracellular multiplication, intracellular spread and infection of adjacent cells, ${ }^{7,8}$ are poorly understood. In the present study the influence of subinhibitory concentrations of antimicrobial agents on the interaction of $S$. flexneri $2 \mathrm{a}$ and $E$. coli $\mathrm{O} 124$ with epithelial cells was determined.

\section{Materials and methods}

\section{Bacterial strains}

Fully virulent wild-type strains of $S$. flexneri 2a (VT100) and E. coli O124 (VT1240) were used. These strains were isolated from patients with dysentery and dysentery-like syndrome in the hospitals of $\mathrm{St}$ Petersburg (Russia) and have been described previously. ${ }^{6}$

\section{Antimicrobial agents}

Chloramphenicol and rifampicin (Sigma), tetracycline (Achromycin; Lederle, New York, NY, USA), and nalidixic acid (Negram; Winthrop, New York, NY, USA) were used for the study. MICs were determined by a broth dilution method with serial two-fold dilutions of the antibiotics in aminopeptide broth and an inoculum of $10^{6} \mathrm{cfu} / \mathrm{ml}$, adjusted spectrophotometrically. The results were read after incubation for $18 \mathrm{~h}$ at $37^{\circ} \mathrm{C}$. Concentrations of antimicrobial agent equivalent to one-third of the MIC $(0.3 \mathrm{mg} / \mathrm{L}$ for rifampicin, chloramphenicol and nalidixic acid and $0.1 \mathrm{mg} / \mathrm{L}$ for tetracycline) were added to the bacterial and tissue culture media.

\section{Culture conditions}

Bacteria were grown in aminopeptide broth or an aminopeptide agar at $37^{\circ} \mathrm{C}$. Before use, bacterial cultures were washed once with phosphate-buffered saline (PBS) and suspended in basal Eagle's medium to 
Table. Virulence factor changes in bacteria cultivated in the presence of subinhibitory concentrations of antimicrobial agents

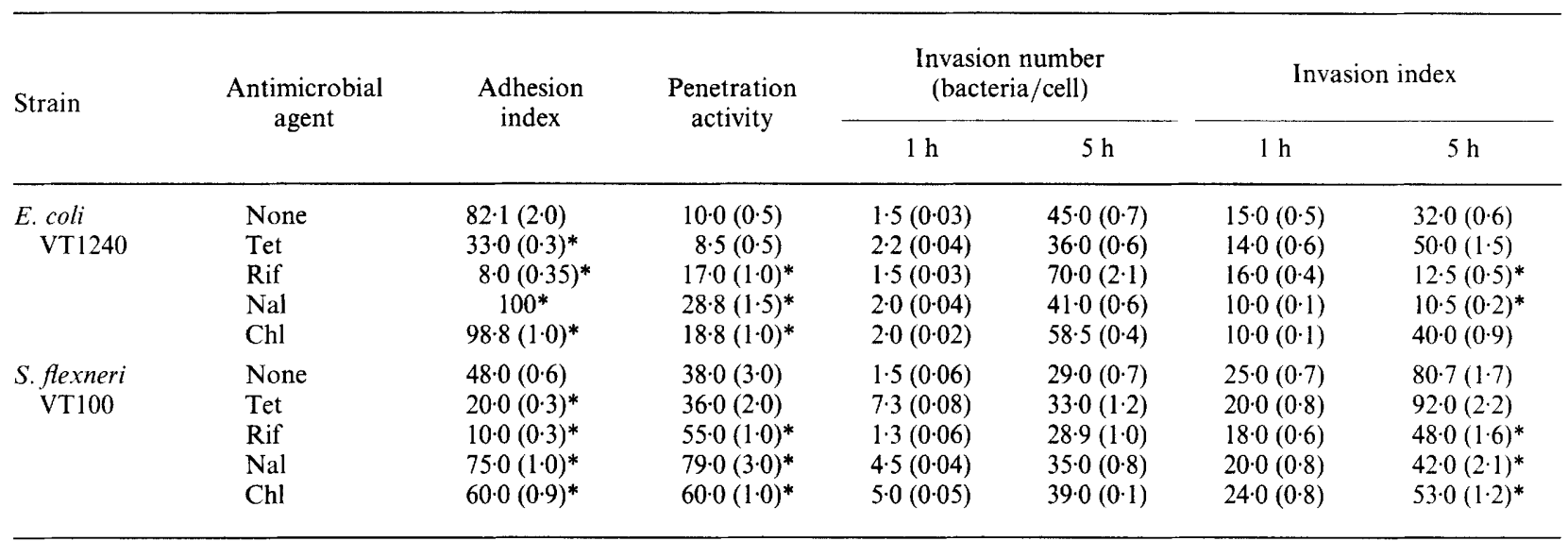

Numbers are the mean (and SD) of five independent experiments.

Tet, tetracycline; Rif, rifampicin; Nal, nalidixic acid; Chl, chloramphenicol.

*Significant differences.

achieve a concentration of $(1-2) \times 10^{9}$ bacteria $/ \mathrm{ml}$. Inocula were standardised according to optical density at $650 \mathrm{~nm}$.

\section{Tissue culture}

HeLa cells were grown in basal Eagle's medium supplemented with heat inactivated fetal calf serum (Flow Laboratories Inc., McLean, VA, USA) $10 \%$.

\section{Assay of virulence factors}

The methods of Sansonetti et al. ${ }^{9}$ and Makino et al. ${ }^{10}$ with some modifications, as described below, were used to test the virulence factors.

For examination of adhesion, HeLa cells were grown on coverslips in glass vials. Samples of bacteria were added to the HeLa cell monolayers to achieve a final concentration of $(1-2) \times 10^{8}$ bacteria $/ \mathrm{ml}$. After incubation for $1 \mathrm{~h}$ at $37^{\circ} \mathrm{C}$ the monolayers were washed eight times with PBS, fixed with methanol and stained with Giemsa solution. The percentage of cells having bacteria on their surfaces (adhesion index) was determined by microscopy. In each test 500 cells were examined.

To study penetration of HeLa cells the coverslip cell cultures were centrifuged at room temperature immediately after addition of the bacteria to sediment the bacteria on the cell surfaces. The coverslips were incubated for $20 \mathrm{~min}$ at $37^{\circ} \mathrm{C}$ to allow penetration of the HeLa cells by the bacteria and were then washed with PBS. Fresh basal Eagle's medium supplemented with gentamicin $100 \mathrm{mg} / \mathrm{L}$ and kanamycin $500 \mathrm{mg} / \mathrm{L}$ was added to kill extracellular bacteria and incubation at $37^{\circ} \mathrm{C}$ was continued for $1 \mathrm{~h}$. Cultures were again washed thoroughly and trypsinised to suspend the cells, which were then counted, disrupted by a solution of sodium deoxycholate $0.5 \%$ in distilled water, serially diluted and plated on nutrient agar to obtain a viable count of the intracellular bacteria. Controls were included in which cells were infected with bacteria, washed and then incubated without genta- micin and kanamycin. Penetration activity was expressed as the number of bacteria in the experimental culture divided by the number in the control and multiplied by 100 . In preliminary experiments, penetration activity of invasive strains of $E$. coli and Shigella spp. (which induce keratoconjunctivitis in the guinea-pig eye) exceeded $5 \cdot 0$, whereas that of noninvasive strains (which did not induce keratoconjunctivitis in guinea-pigs) was below 2.0.

To estimate intracellular multiplication and spread to adjacent cells, the bacteria were sedimented on to the coverslip cultures of HeLa cells, incubated for $20 \mathrm{~min}$ at $37^{\circ} \mathrm{C}$ and washed. Fresh basal Eagle's medium with gentamicin and kanamycin was added and, after incubation for $5 \mathrm{~h}$, the cells were fixed and stained as described above. The invasion number was assessed as the number of bacteria/HeLa cell and the invasion index was expressed as the relative number of infected HeLa cells after growth in the presence of antibiotics for $1 \mathrm{~h}$ and $5 \mathrm{~h}$. The multiplication of bacteria in these conditions was assumed to be possible only inside the HeLa cells, and the increase in the number of infected cells to result only from the spreading of bacteria from one cell to another.

In all experiments, bacteria were added to $\mathrm{HeLa}$ cells at the same time as antimicrobial agent. Control cultures without antibiotic were studied in parallel to the treated ones.

The paired $t$ test $(\mathrm{p}=0.05)$ was used to compare the virulence factor values in the presence and absence of subinhibitory concentrations of antibiotic.

\section{Results and Discussion}

The effects of subinhibitory concentrations of tetracycline, rifampicin, nalidixic acid and chloramphenicol on the activity of different virulence factors of $S$. flexneri 2a (VT100) and E. coli O124 (VT1240) are shown in the table. Bacterial adhesion was highly sensitive to concentrations of antimicrobial agents 
below the MIC. All the agents used inhibited or potentiated this stage of the host-parasite interaction. Tetracycline and rifampicin inhibited adhesion of both strains. In the presence of rifampicin the adhesion index of $E$. coli VT1240 was reduced by $90 \%$ and that of $S$. flexneri VT100 by $70 \%$. In contrast, nalidixic acid and chloramphenicol potentiated adhesion. In the presence of these agents $E$. coli VT1240 adhered to practically all the HeLa cells in monolayers. The penetration of bacteria into the HeLa cells was increased by all the antimicrobial agents used, except tetracycline. In the presence of nalidixic acid penetration was enhanced three-fold.

The process of intracellular multiplication was minimally affected by concentrations of antimicrobial agents below the MIC, but infection of adjacent cells was significantly reduced by rifampicin and nalidixic acid. These agents almost abolished the ability of $E$. coli VT1240 to spread to adjacent cells, while $S$. flexneri VT100 was affected to a lesser extent. Tetracycline and chloramphenicol did not suppress the process of infection of adjacent cells.

It has been reported that Shigella strains may lose

\section{References}

1. Shibl AM. Influence of antibiotics on host-parasite interactions with an emphasis on in-vivo studies. J Chemother $1990 ; 2$ : 75-78.

2. Tetz VV, Borysov LB. Effect of antibiotics on bacterial population variability. Antibiot Chemother 1988; 4: 299-303 (in Russian).

3. Eisenstein BI, Beachey EH, Ofek I. Influence of sublethal concentrations of antibiotics on the expression of the mannose-specific ligand of Escherichia coli. Infect Immun $1980 ; 28$ : 154-159.

4. Schifferli DM, Beachey EH. Bacterial adhesion: modulation by antibiotics which perturb protein synthesis. Antimicrob Agents Chemother 1988; 32: 1603-1608.

5. Tetz VV, Rybalchenko OV, Savkova GA. Surface films of Escherichia colonies. FEMS Microbiol Lett 1993; 107: 231-240.

6. Tetz VV, Rybalchenko OV, Savkova GA. Ultrastructure of the surface film of bacterial colonies. J Gen Microbiol 1993; 139: $855-858$. virulence plasmids in the presence of subinhibitory concentrations of antibiotics, ${ }^{11}$ but in our experiments the strains used for the study appeared to retain their virulence plasmids, since proteins that determine the ability to invade are coded by plasmid genes. ${ }^{9}$

Each of the bacterial virulence factors examined is a necessary component in the development of the diseases caused by enteroinvasive $E$. coli and $S$. flexneri. ${ }^{7-10}$ The results of this study indicate that expression of some invasive enterobacterial virulence factors may be influenced by antimicrobial agents at concentrations which do not inhibit bacterial growth. The most significant changes were observed in the processes of adhesion and penetration and to a lesser extent in the infection of adjacent cells. Intracellular multiplication was not affected. In the experimental conditions used, nalidixic acid and chloramphenicol enhanced the adhesive and penetrative activities of the bacteria tested, and nalidixic acid (but not chloramphenicol) also inhibited the re-infection process.

The ability of chloramphenicol to increase bacterial virulence in vitro in the HeLa cell model was the most general effect observed in this study.

7. Hale TL, Bonventre PF. Shigella infection of Henle intestinal epithelial cells: role of the bacterium. Infect Immun 1979; 24: 879-886.

8. Hale TL, Formal SB. Protein synthesis in HeLa or Henle 407 cells infected with Shigella dysenteriae 1, Shigella fexneri 2a, or Salmonella typhimurium W118. Infect Immun 1981: 32: $137-144$.

9. Sansonetti PJ, Hale TL, Demmin GJ, Kapfer C, Collins HH, Formal SB. Alterations in the pathogenicity of Escherichia coli $\mathrm{K}-12$ after transfer of plasmid and chromosomal genes from Shigella flexneri. Infect Immun 1983; 39: 1392-1402.

10. Makino S, Sasakawa C, Kamata K, Kurata T, Yoshikawa M. A genetic determinant required for continuous reinfection of adjacent cells on large plasmid in S. flexneri 2a. Cell $1986 ; 46$ : 551-555.

11. Michel-Briand Y, Laporte JM, Couetdic G, Sansonetti PJ. Elimination of a virulence plasmid from Shigella sonnei and Escherichia coli by antibiotics. Ann Inst Pasteur Microbiol 1986; 137B: 291-295. 